\title{
Point-of-care ultrasound (PoCUS) in the early diagnosis of novel coronavirus 2019 disease (COVID-19) in a first-level emergency department during a SARS-CoV-2 outbreak in Italy: a real-life analysis
}

\author{
Simone Bianchi ${ }^{1,5}$ (1) . Caterina Savinelli ${ }^{1}$ - Elisa Paolucci ${ }^{2}$. Lorenzo Pelagatti ${ }^{2}$ - Erica Sibona ${ }^{2} \cdot$ Natalia Fersini $^{2}$. \\ Michele Buggea ${ }^{2}$. Camilla Tozzi ${ }^{1}$ - Germana Allescia ${ }^{3} \cdot$ Diana Paolini $^{4} \cdot$ Michele Lanigra $^{1}$
}

Received: 12 October 2020 / Accepted: 13 January 2021 / Published online: 21 April 2021

(c) Società Italiana di Medicina Interna (SIMI) 2021

\begin{abstract}
In December 2019, the severe acute respiratory syndrome coronavirus 2 (SARSCoV-2) spread worldwide, challenging emergency departments (EDs) with the need of rapid diagnosis for appropriate allocation in dedicated setting. Many authors highlighted the role of lung ultrasound (LUS) in management of the novel coronavirus disease 2019 (COVID-19). The study aims to analyze the performance of LUS in the early identification of COVID-19 patients in ED during a SARS-CoV-2 outbreak. We prospectively collected consecutive adult patients admitted to a first-level ED in Powered by Editorial Manager ${ }^{\circledR}$ and ProduXion Manager ${ }^{\circledR}$ from Aries Systems Corporation Florence with history or symptoms suggestive for COVID-19 that underwent LUS during the ED management. LUS findings were categorized in 6 discrete main etiological patterns. "A", "Cardiogenic B" and "Typical C" patterns were referred as non-COVID-19-suggestive, while "Atypical" B or C patterns, "Multiple Consolidations" pattern and "ARDS" pattern were referred as COVID-19-suggestive. The primary outcome was the diagnosis of SARS-CoV-2 infection. From 12 March to 12 May 2020, 360 patients were enrolled. COVID-19 suggestive LUS findings were significantly associated with final COVID-19 diagnosis (86\% in COVID-19 vs 29\% in non-COVID-19, $p<0.001)$. The presence in ED of at least one in positive swab OR a COVID-19-suggestive LUS showed a sensitivity of $97 \%$ and a negative predictive value (NPV) of 98\%. In patients with known SARS-CoV-2 exposition in the last 14 days, a COVID-19-suggestive pattern at LUS had a positive predictive value (PPV) of 97\% for COVID-19 diagnosis. Point-of-care ultrasound (PoCUS) is a valuable tool for diagnostic stratification during COVID-19 outbreaks. LUS can help physicians in identifying false-negative RT-PCR, improving its diagnostic sensitivity in ED.
\end{abstract}

Keywords Point-of-care ultrasound $\cdot$ Lung ultrasound $\cdot$ Emergency department $\cdot$ COVID- $19 \cdot$ SARS-CoV-2

Simone Bianchi

simone.bianchi@uslcentro.toscana.it

1 Emergency Department, Santa Maria Nuova Hospital, Middle-Tuscany Local Health Agency, Florence, Italy

2 Emergency Department, Careggi University Hospital, Florence, Italy

3 Radiology and Diagnostic Imaging Department, Santa Maria Nuova Hospital, Middle-Tuscany Local Health Agency, Florence, Italy

4 Health Management Unit, Careggi University Hospital, Florence, Italy

5 Emergency Department, Ospedale Santa Maria Nuova, Azienda Unità Sanitaria Locale Toscana Centro, Florence, Italy

\section{Introduction}

In December 2019, a new coronavirus family, now named severe acute respiratory syndrome coronavirus 2 (SARSCoV-2), was found in the city of Wuhan, in China's Hubei province. Within a few months from the first report, it has spread rapidly worldwide, assuming pandemic characteristics.

The novel coronavirus disease 2019 (COVID-19) is characterized by a wide range of signs and symptoms ranging from asymptomatic or poorly symptomatic cases to severe pneumonia and, in about $20 \%$ of infected patients, acute respiratory distress syndrome (ARDS) [1, 2], with high morbidity and mortality.

The gold standard for SARS-CoV-2 infection diagnosis is the real-time reverse transcription polymerase chain reaction 
(RT-PCR), mainly performed on naso-pharyngeal swab. RTPCR showed a suboptimal sensitivity, and infection should not be ruled out on the basis of RT-PCR alone [3-5]. Two main strategies were suggested to deal with the high rates of false-negative RT-PCR: multiples swabs can improve the sensitivity of the test, and, as suggested by many authors, the routine use of chest computed tomography (CT) or lung ultrasound (LUS) among with clinical and blood exams in patients with suspected COVID-19 [6, 7].

Chest CT has often been chosen as the routine imaging technique for diagnosis and monitoring of COVID-19. Patients with confirmed COVID-19-related pneumonia showed characteristic CT features like unilateral or bilateral ground glass opacities (GGO), which refers to areas of misty pulmonary opacity with conservation of parenchymal architecture, reticular pattern, crazy-paving pattern, lung consolidations, pleural abnormalities, such as thickening and effusion, airway abnormalities including bronchiectasis and bronchial wall thickening, air bronchogram and lymphadenopathy (Fig. 1) [8-12].

LUS may support the identification and the clinical management of patients with COVID-19. The main abnormalities found at LUS in patients with COVID-19 are dense or confluent B lines, small multiple subpleural consolidations and pleural line abnormalities. "White lung" areas with confluent and melted B lines and absence of A lines can be seen in severe cases. Most patients showed bilateral involvement. Large consolidations with air bronchogram and pleural effusions were usually associated with alternative diagnosis [13]. A challenging sonographic differential diagnosis is between interstitial B pattern and cardiogenic pulmonary oedema. An integrated bedside point-of-care ultrasound (PoCUS) assessment performed by emergency physicians (EPs) can help to determine interstitial syndrome etiology. Homogeneous distribution of B lines, smooth pleura, intact sliding, pleural effusions, inferior vena cava (IVC) dilatation with absence of respiratory variations and left ventricular dysfunction are usually present in cardiogenic lung congestion, while irregular multifocal B lines distribution, subpleural consolidations and spared areas are suggestive for lung interstitial diseases, like COVID-19 [14].

Many authors highlighted the role of LUS in field and hospital management and in identifying lung involvement in patients concerning SARS-COV2 infection and whom RT-PCR and radiological findings are inconclusive [15-18].

\section{Aim of the study}

The study aims to analyze the performance of PoCUS in the early identification of COVID-19-related pneumonia in a population of patients referring to a first-level italian ED during a SARS-CoV-2 outbreak.

\section{Materials and methods}

\section{Setting}

In Italy, during COVID-19 outbreak, dedicated pathways to confirmed or suspected COVID-19, defined as "COVID-19 pathway", were identified in all hospitals. In particular, all patients admitted to the emergency department (ED) underwent a Pre-Triage outside the ED, and patients who met inclusion criteria for COVID-19-suspicion were sent by a dedicated pathway in a physically separated section of the ED with dedicated personnel and equipment. The criteria for the "COVID-19-pathway" included patients referring to the ED with known history of high risk contact with a COVID19 patient, or either one among cough, fever or shortness of breath of any cause in the last 14 days and patients with outof-hospital diagnosis of pneumonia (even if asymptomatic) whom were sent in ED for further evaluation.

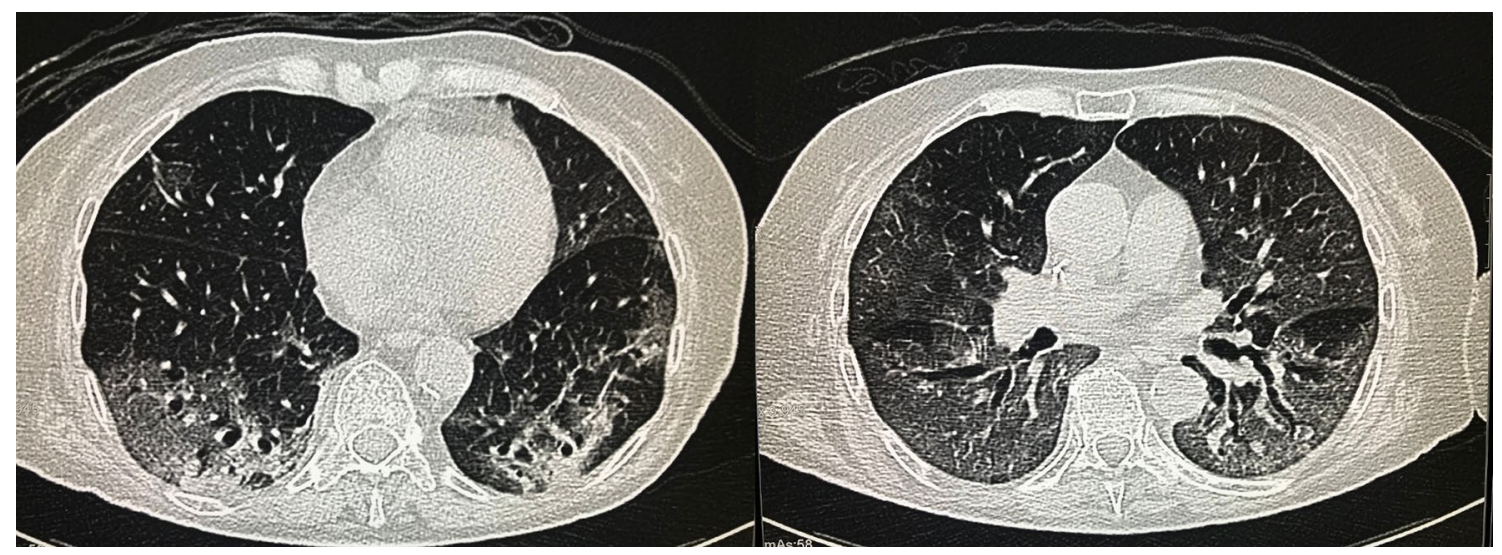

Fig. 1 Chest CT scan showing ground glass opacities (left) and crazy-paving pattern (right) 


\section{Study design}

The study was a single-center prospective cohort study conducted in a first-level, urban ED with an approximate annual volume of 38,000 visits/year. We prospectively collected consecutive adult, nonpregnant patients admitted to COVID-19 pathway of the ED of Santa Maria Nuova Hospital in Florence, who underwent LUS during the ED. We selected a threshold age of 16 years old due to the Central Tuscany Emergency Service centralization protocols: in absence of contraindications (for instance, the need for emergency time-dependent treatments), trauma patients under 14 years of age are primarily admitted to the local children hospital, as well as non-traumatic patients under the age of $16[19,20]$.

Inclusion criteria (at least one):

high-risk exposition to known COVID-19 case according to the World Health Organization (WHO) definitions [21] in the last 14 days;

at least one clinical criteria for SARS-CoV-2 suspected infection in the last 14 days, namely: cough, fever, or shortness of breath;

any radiological finding of pneumonia.

Additional mandatory inclusion criteria:

at least one LUS evaluation in ED.

Exclusion criteria:

age $<16$ years;

pregnancy;

known SARS-CoV-2 active infection. Patients with known positive RT-PCR for SARS-CoV-2 were considered actively infected until demonstration of 2 consecutive negative RT-PCR testing on naso-pharyngeal swab at $48 \mathrm{~h}$ according to WHO definition at enrollment time [22].

The study was conducted in accordance with the 1964 Helsinki declaration and its later amendments, and was approved by the institutional review board (protocol CEAVC 17260). Each patient gave informed consent to to take part in the study and agreed to the publication of their personal data. An agreement with their legal representative or guardian was executed for patients legally or physically unable to give informed consent.

Patients data were anonymized and collected in a strictly confidential electronic database according to national and international privacy laws.

\section{Sample size calculations}

The aim of the study was to analyze the diagnostic power of LUS in patients admitted to the ED with suspicion of COVID-19 infection. When the study was designed the known prevalence of COVID-19 in Tuscany was $0.0016 \%$ [8]. Considering a suspected high prevalence of asymptomatic patients, we estimated a 20 -times higher real prevalence $(0.032 \%)$. With a desired statistical power of $95 \%$, a supposed prevalence of COVID-19 in the study population of $10 \%$ and an alpha error of 0.05 we estimated a sample size of 310 patients. The final study population counted 370 patients with a 39\% of COVID-19 prevalence and accomplished the requests for statistical analysis.

\section{Patients and data sources}

We prospectively collected and reviewed the clinical records of eligible patients. We registered demographic characteristics of patients, such as age, sex, comorbidities, home therapy, vital signs, triage priority code, blood and imaging tests performed (including venous blood samples, arterial blood gas analysis, SARS-CoV-2 antibodies, LUS, chest $\mathrm{X}$-ray and chest computed tomography $[\mathrm{CT}]$ ), respiratory support therapy (including oxygen delivery, non-invasive or invasive mechanical ventilation).

Tuscany EDs use a five-level triage system, which is divided into five code (no urgent matter, evaluation within $240 \mathrm{~min}$ ), four code (minor urgency, evaluation within $120 \mathrm{~min}$ ), three code (standard urgency, evaluation within $60 \mathrm{~min}$ ), two code (severe illness or injury, evaluation within $15 \mathrm{~min}$ ) and one code (life threatening, immediate treatment) [23]. Similar five-graded triage systems, i.e. Manchester Triage System (MTS) [24] can be found in literature.

Data on symptoms, time from onset, comorbidities and pharmacological treatment were self-reported and confirmed after reviewing clinical charts. Cardiovascular diseases were defined as known coronary artery diseases or previous ischaemic stroke. Siddiqi et al. proposed a 3 -class clinical staging of COVID-19 clinical patterns [25]. Patients were categorized according to Thoracic Ultrasound Academy (AdET) severity classification for its better discrimation of patients medical needs (home treatment and follow-up vs hospital admission vs intensive care unit (ICU) needs). Clinical patterns were lately grouped according to the WHO definition in mild, moderate, severe and critical, as shown in Table 1 [26-28]. SARS-CoV-2 swabs were collected by trained physicians or professional nurses and analyzed in the nearest laboratory licensed by National Health Authority for SARS-CoV-2 testing (Careggi University Hospital Laboratory, Florence). An expert radiology specialist blinded from clinical data revised chest CT scan images using a viewing console. GGO were 
Table 1 Patient's clinical phenotype $[25,26,30]$

\begin{tabular}{llll}
\hline AdET [26] & $\begin{array}{l}\text { Siddiqi et.al } \\
\text { [27] }\end{array}$ & WHO [28] & Clinical features \\
\hline 0 & I & - & \\
1 & I & Mild & $\begin{array}{l}\text { Asymptomatic patient } \\
\text { Fever (with or without respiratory symptoms), no } \\
\text { signs of pneumonia, no hypoxia } \\
\end{array}$ \\
2 & II & Moderate & Pneumonia without hypoxia \\
3 & III & Severe & Pneumonia and tachypnea/hypoxia \\
4 & III & Critical & Pre-ARDS, non-invasive mechanical ventilation need \\
5 & III & Critical & ARDS or mechanical ventilation or ICU admission \\
\hline
\end{tabular}

AdET Thoracic ultrasound academy; WHO World Health Organization; ARDS acute respiratory distress syndrome; $I C U$ intensive care unit defined as hazy increased lung attenuation with preserved bronchial and vascular margins, while consolidations were defined as opacities involving vessels and airways walls [27]. Different scenarios have been found and classified as COVID-19 suggestive or not suggestive. Multiple, bilateral, peripherally distributed GGO with crazy-paving pattern in absence of other non-COVID-suggestive lesions (as pleural effusion or lymphadenopathy) were considered as COVID-19 suggestive. GGO with atypical distribution for COVID-19 (not bilateral, not peripheral) or associated with lesions suggestive for different diagnosis were defined as COVID-19 compatible but non typical (nondiagnostic exam). Images without criteria for COVID-19, nonpathologic images and eventually images suggestive for alternative diagnosis alone (blooming tree or excavated lesions, large consolidation with air bronchogram, etc.) were considered as COVID-19 not suggestive. For sensitivity analysis COVID-19 possible but not typical images were considered as COVID-suggestive.

To avoid compilation bias, different researchers were involved in patient management, data collection, followup and statistical analysis. Moreover, PoCUS data were immediately reported in a clinical chart by the sonographer, and prospectively collected at enrollment time before the availability of swabs and follow-up results, to grant researchers blinding and to prevent observer bias.

\section{Point-of-care ultrasound (PoCUS)}

PoCUS were performed by physicians with expertise in LUS, proved by completion of a LUS training course accredited by the Italian Society of Emergency Medicine (SIMEU) or by the Italian Society of Medical and Biology Ultrasound (SIUMB), and performance and interpretation of at least 50 LUS examinations. A preliminar collegial theoretical-practical briefing was performed among investigators before enrollment, to standardize the technique of execution and the LUS report, with special attention for posterior fields scan and pleural line abnormalities description. LUS scans were performed with linear or convex probes according to body size. A complete 12-field LUS [28] was performed when possible (Figs. 2, 3). In obese uncompliant patients that could not be moved anterior and lateral ultrasound scansions were performed, together with at least a partial view of the posterior basal areas. IVC size and respiratory variations and LV function were eventually evaluated with a sectorial probe according to clinician needs for a correct PoCUS evaluation, particularly for interstitial syndrome
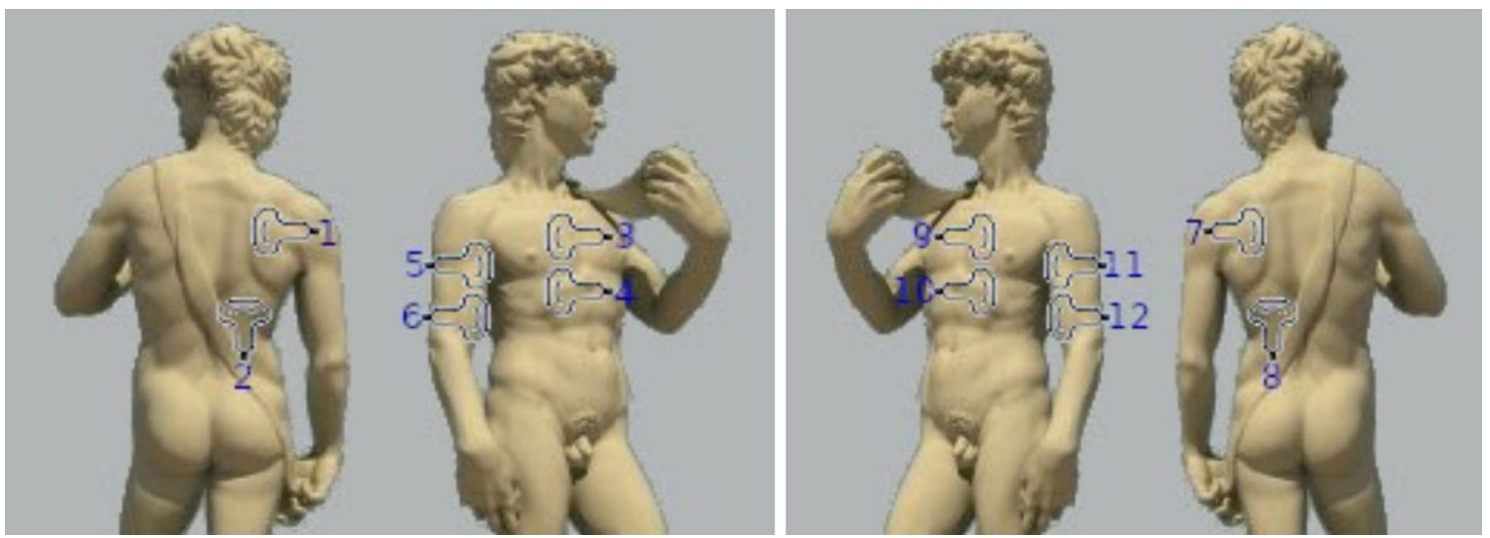

Fig. 2: LUS evaluation windows 

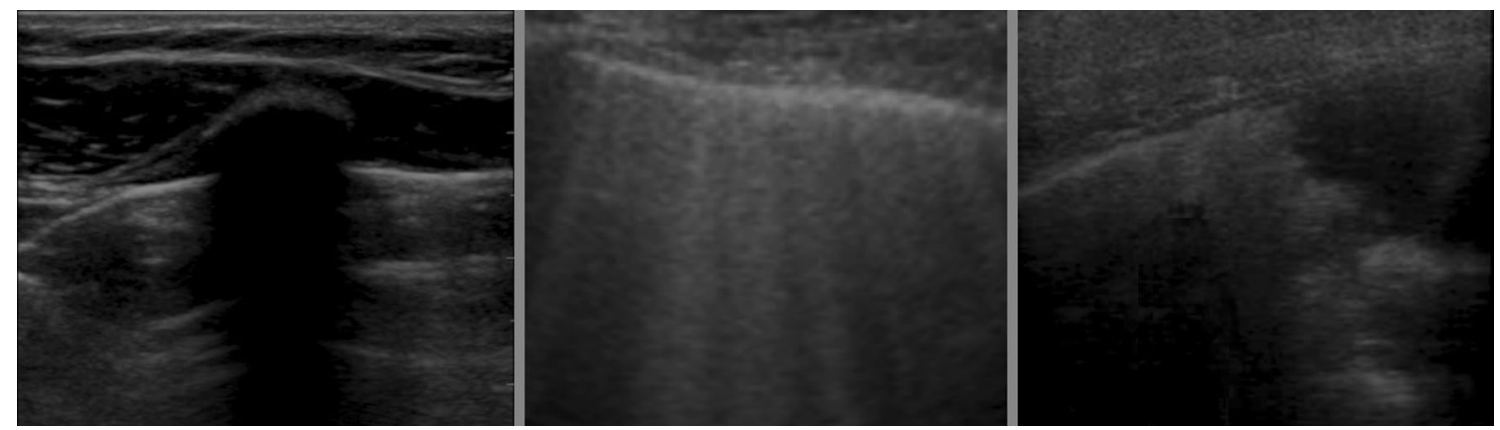

Fig. 3 LUS showing a pattern (left), b pattern (middle), lung consolidation (c pattern, right)

differential diagnosis. Ultrasound devices complete sterilization with alcoholic solution was performed before and after every use. As since the beginning of disease, even if similarly to other interstitial pneumonia, COVID-19 pneumonia shows uncommon LUS findings compared to those seen in common practice, POCUS findings were detailed in clinical charts and categorized in six discrete main etiological patterns as reported in Table 2. "A", "Cardiogenic B" and "Typical C" patterns were referred as COVID-19 not suggestive, while "Atypical" B or C patterns, "Multiple Consolidations" pattern and "ARDS" pattern were referred as COVID-19 suggestive. Some explanatory examples are given in Figs. 4 and 5.

\section{Endpoint}

The primary outcome was the diagnosis of SARS-CoV-2 infection according to WHO definitions at any time during hospital management or at clinical follow-up. All RT-PCR positive cases were considered true positive [21].

\section{Follow-up}

Clinical follow-up was routinely performed at 28-days from hospital discharge by reviewing the ED archives and other medical charts; a phone follow-up was also performed when needed. In case of nonconclusive diagnosis, a further followup was subsequently performed.

\section{Statistical analysis}

Continuous variables were reported as mean \pm standard deviation (SD) or medians and interquartile ranges (IQR), while categorical variables were reported as counts and percentages. Statistical comparisons of demographic and clinical features were performed using the $\chi^{2}$ test and Pearson's exact test for categorical variables (with normal and nonnormal distribution, respectively), whereas the Student's $t$ test and Fisher exact test were used for continuous variables. $p$ values $<0.05$, from a two-sided test, were considered to indicate statistical significance.

Table 2 PoCUS etiological patterns and interpretation

\begin{tabular}{lll}
\hline Pattern & LUS findings & Interpretation \\
\hline A & $\begin{array}{l}\text { Normal LUS: A lines, absence of significative B lines (<3/field), absence of lung } \\
\text { consolidations }\end{array}$ & Alternative diagnosis suggestive \\
Cardiogenic B & Heart failure suggestive B lines: diffuse homogeneous B pattern with base-apical & \\
& typical progressive distribution, regular pleural line, eventually with enlarged \\
& inferior vena cava and/or left ventricular dysfunction and/or pleural effusion (when & \\
& assessed) & \\
Pypical C & Presence of a single large basal lung consolidation, eventually with air bronchogram & \\
and/or focal perilesional interstitial syndrome & COVID-19 suggestive \\
Atypical B/C & Presence of apical lung consolidations and/or atypical distribution of B lines (for & \\
& example non-homogeneous distribution with blurred pleural line, focal apical B & \\
lines, eventually with inferior vena cava collapsibility and absence of pleural effu- & \\
Multiple consolidations & Multiple small lung consolidations, with or without focal perilesional B pattern \\
ARDS & Multiple lung consolidations and white lung and/or bilateral multifocal B pattern \\
& with shattered areas of A pattern & \\
\hline
\end{tabular}

LUS Lung ultrasound; COVID-19 SARS-CoV-2 disease; ARDS acute respiratory distress syndrome 


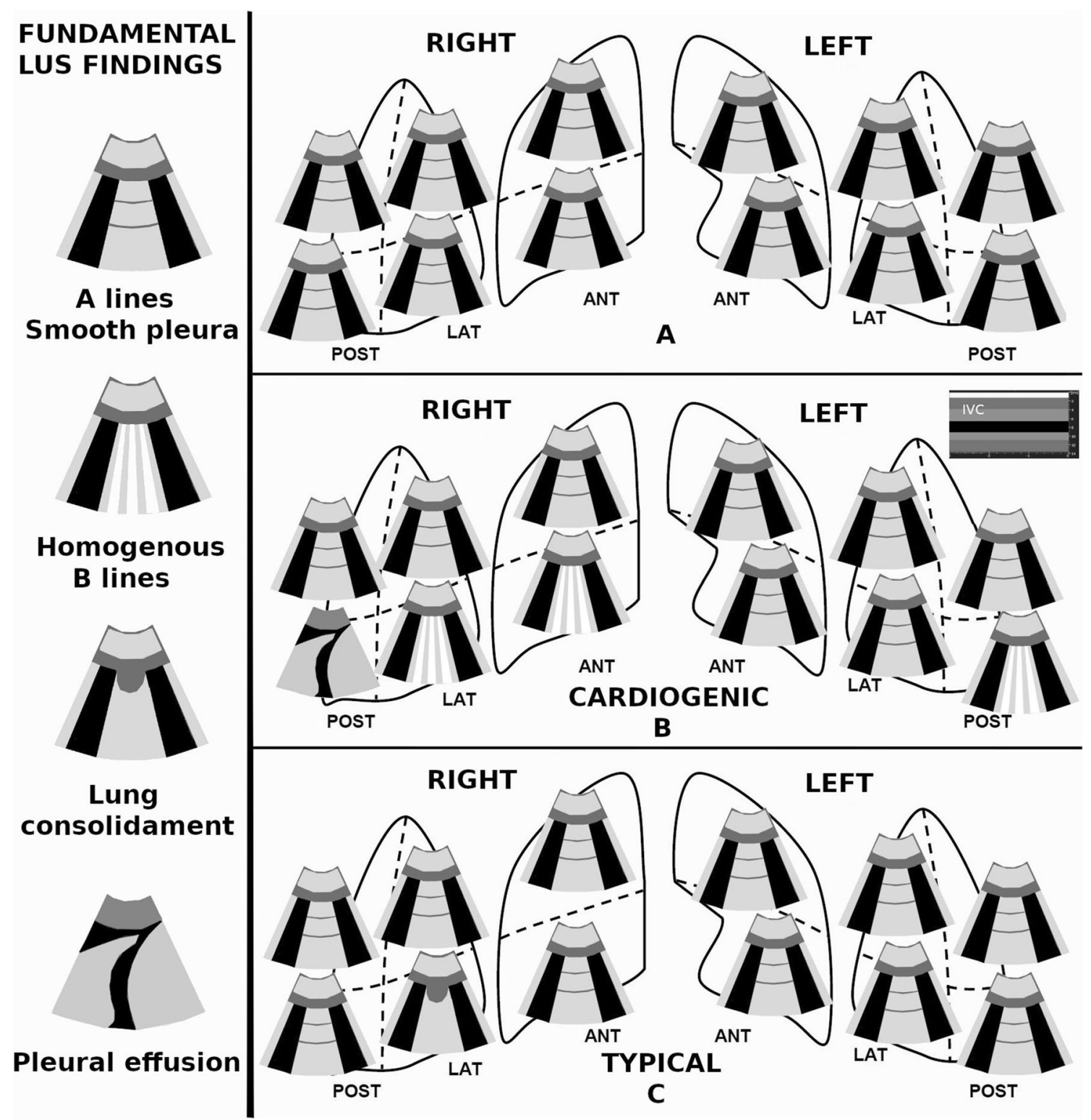

Fig. 4 Examples of LUS patterns not suggestive for COVID-19. Post posterior; Lat lateral; ant anterior; IVC inferior vena cava

Using RT-PCR results as reference, sensitivity, specificity, positive predictive value (PPV), negative predictive value (NPV), and accuracy of diagnostic testing were calculated.

Cohen's kappa were estimated for evaluation of interrater reliability for LUS findings.

Calculations were performed by a dedicated researcher that was not involved in data collection or patient management. SPSS version 24 (SPSS Inc., Chicago, Illinois, USA) was used for all analysis.

\section{Main results}

From 12th March to 12th May 2020, during a COVID19 outbreak, 474 patients were admitted to Santa Maria Nuova ED COVID-19 pathway. In 100 patients LUS was not feasible, not performed, not adequate or not reported for revision. Most of these patients were asymptomatic and were referred to ED only to perform naso-pharyngeal swab because they were close contact to COVID-19 cases. Eight 


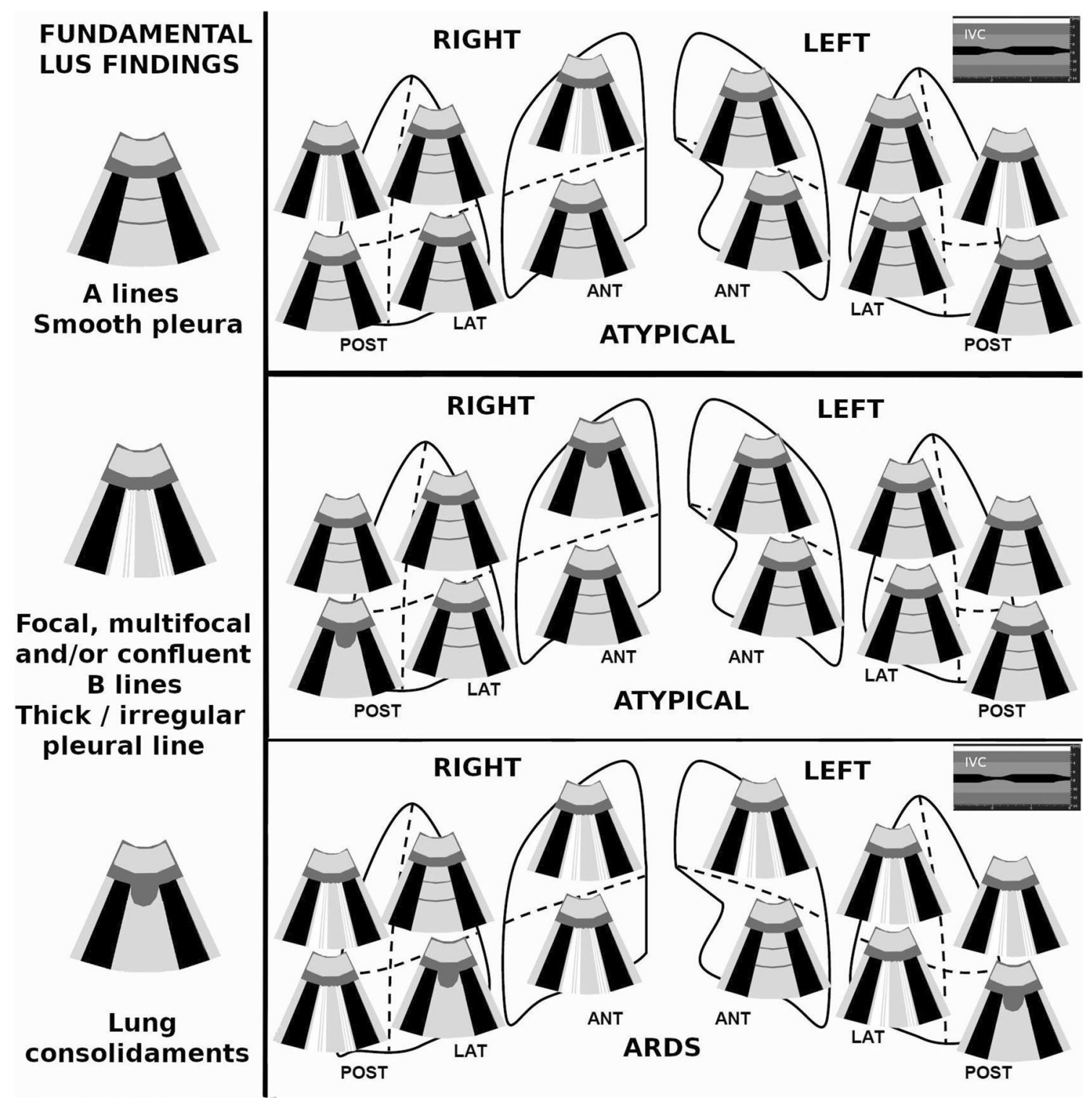

Fig. 5 Examples of LUS patterns suggestive for COVID-19. Post posterior; Lat lateral; Ant anterior; IVC inferior vena cava; ARDS acute respiratory distress syndrome

patients could not be enrolled because they denied their consent to study participation. Three hundred and sixtysix patients were finally enrolled. Six patients $(1,6 \%)$ were lost to follow-up and excluded from analysis. The study population counted 360 patients, median age was 72 years (IQR 51-85), 73 years (IQR 60-82) in COVID-19 and 71 years (IQR 44-85) in non-COVID-19). Most patients (70\%) had standard urgent symptoms at Triage, 19\% had severe or life-threatening symptoms, and $11 \%$ had minor or non-urgent matters. No differences were shown between COVID-19 and non-COVID-19 groups ( $p=$ NS). Median onset-to-ED admission time was 3 days (IQR 1-7), no differences were shown according to final COVID-19 diagnosis (5 [IQR 2-10] in COVID-19 vs 3 [IQR 1-7] in non-COVID-19, $p=$ NS). More than half of enrolled patients $(n=240,67 \%)$ were admitted to ED within 5 days from symptom onset. Baseline population characteristics are shown in Tables 3 and 4 . Overall 28-days mortality was $16 \%$ (24\% in COVID-19 vs $12 \%$ in non-COVID-19, $p=0.001$ ). A diagnosis of COVID-19 was performed in 21 (18\%) patients with first negative RT-PCR testing in ED. Overall RT-PCR sensitivity was 85\% (95\% CI 79-91\%). In subgroups analysis, false-negative RT-PCR were not related with days from symptoms onset $(20 \%$ in asymptomatic group vs $21 \%$ in $0-3$ days vs $18 \%$ in $4-6$ days vs $14 \%$ in $7-9$ days vs $5 \%$ in $10-12$ days vs $17 \%$ in $>12$ days group, $p=\mathrm{NS}$ ). False-negative first RT-PCR was found in $24 \%$ of mild COVID-19, in $13 \%$ of moderate COVID-19 
Table 3 Population baseline characteristics

\begin{tabular}{lcccr}
\hline & Total $(n=360)$ & $\begin{array}{l}\text { Not COVID-19 } \\
(n=220)\end{array}$ & COVID-19 $(n=140)$ & $p$ \\
\hline Male sex & $180(50 \%)$ & $77(55 \%)$ & $103(45 \%)$ & 0.146 \\
COPD & $55(15 \%)$ & $34(14 \%)$ & $21(15 \%)$ & 0.453 \\
Cardiovascular disease & $44(12 \%)$ & $35(16 \%)$ & $9(6,4 \%)$ & 0.005 \\
Active smoking & $52(14 \%)$ & $46(21 \%)$ & $6(4,3 \%)$ & $<0.001$ \\
Obesity & $25(6,9 \%)$ & $21(10 \%)$ & $4(2,9 \%)$ & 0.015 \\
Diabetes & $52(14 \%)$ & $32(15 \%)$ & $20(14 \%)$ & 0.972 \\
Immunodeficit & $7(1.9 \%)$ & $4(1.8 \%)$ & $3(2.1 \%)$ & 0.833 \\
Hypertension & $145(41 \%)$ & $89(41 \%)$ & $56(40 \%)$ & 0.655 \\
Chronic kidney disease & $40(11 \%)$ & $24(11 \%)$ & $16(11 \%)$ & 0.973 \\
Neoplastic disease & $27(7.5 \%)$ & $15(6.8 \%)$ & $12(8.6 \%)$ & 0.214 \\
DNR conditions & $36(10 \%)$ & $18(8.2 \%)$ & $18(13 \%)$ & 0.154 \\
Known COVID-19 contact & $59(16 \%)$ & $15(6.8 \%)$ & $44(31 \%)$ & $<0.001$ \\
Nursing home resident & $59(16 \%)$ & $17(7.7 \%)$ & $42(30 \%)$ & $<0.001$ \\
\hline
\end{tabular}

COPD Chronic obstructive pulmonary disease; NR do not resuscitate; COVID-19 SARS-CoV-2 infection disease

\begin{tabular}{|c|c|c|c|c|}
\hline & Total $(n=360)$ & $\begin{array}{l}\text { Not COVID-19 } \\
(n=220)\end{array}$ & COVID-19 $(n=140)$ & $p$ \\
\hline \multicolumn{5}{|c|}{ Triage priority (code) } \\
\hline Urgent (1-2) & $70(19 \%)$ & $43(19 \%)$ & $27(19 \%)$ & \multirow[t]{3}{*}{0.132} \\
\hline Differible (3) & $250(70 \%)$ & $144(64 \%)$ & $106(76 \%)$ & \\
\hline Minor (4-5) & $40(11 \%)$ & $33(15 \%)$ & $7(5,0 \%)$ & \\
\hline \multicolumn{5}{|c|}{ Symptoms (multiple symptoms possible for each patient) } \\
\hline Fever & $241(67 \%)$ & $130(59 \%)$ & $111(79 \%)$ & $<0.001$ \\
\hline Cough & $103(29 \%)$ & $55(25 \%)$ & $48(34 \%)$ & 0.057 \\
\hline Dyspnea & $100(28 \%)$ & $66(30 \%)$ & $34(24 \%)$ & 0.238 \\
\hline Sore throat & $9(2.5 \%)$ & $8(3.6 \%)$ & $1(0.7 \%)$ & 0.083 \\
\hline Hemoptysis & $4(1.1 \%)$ & $2(0.9 \%)$ & $2(1.4 \%)$ & 0.647 \\
\hline Enteritis & $16(4.4 \%)$ & $11(5.0 \%)$ & $5(3.6 \%)$ & 0.521 \\
\hline Neurological & $15(4.2 \%)$ & $14(6.4 \%)$ & $1(0.7 \%)$ & 0.009 \\
\hline \multicolumn{5}{|c|}{ AdET [26] (WHO [28]) Clinical phenotype } \\
\hline Asymptomatic & $10(2.8 \%)$ & $8(3.6 \%)$ & $2(1.4 \%)$ & \multirow[t]{6}{*}{$<0.001$} \\
\hline 1 (mild) & $94(26 \%)$ & $79(36 \%)$ & $15(11 \%)$ & \\
\hline 2 (moderate) & $122(34 \%)$ & $73(33 \%)$ & $49(35 \%)$ & \\
\hline 3 (severe) & $88(24 \%)$ & $40(18 \%)$ & $48(34 \%)$ & \\
\hline 4 (critical) & $31(8.6 \%)$ & $15(6.8 \%)$ & $16(11 \%)$ & \\
\hline 5 (critical) & $15(4.2 \%)$ & $5(2.3 \%)$ & $10(7.1 \%)$ & \\
\hline
\end{tabular}

AdET Accademia di Ecografia Toracica; WHO World Health Organization; COVID-19 SARS-CoV-2 infection disease

Table 4 Symptoms and severity ad ED admission (mean Cohen's kappa $0.74, p<0.001$ ), but a very good correlation for the final COVID-19-suggestive interpretation according to the study protocol (mean Cohen's $k$ $0.80, \mathrm{p}<0.001$ ). LUS patterns and TC results according to COVID-19 diagnosis are reported in Table 5.

A complete 12-field LUS was performed in 298 patients (83\%). COVID-19 suggestive LUS findings were 
Table 5 Imaging results according to COVID-19 infection

\begin{tabular}{llcc}
\hline & Total & Not COVID-19 \\
$n=360$ & $n=220$ & COVID-19 \\
LUS & $107(30 \%)$ & $95(43 \%)$ & 140 \\
\hline A pattern & $46(13 \%)$ & $43(20 \%)$ & $3(2.1 \%)$ \\
Cardiogenic B pattern & $24(6.7 \%)$ & $19(8.6 \%)$ & $5(3.6 \%)$ \\
Typical C pattern & $63(18 \%)$ & $34(16 \%)$ & $29(21 \%)$ \\
Atypical pattern & $8(2.2 \%)$ & $2(0.9 \%)$ & $6(4.3 \%)$ \\
Multiple lung consolidations & $112(31 \%)$ & $27(12 \%)$ & $85(61 \%)$ \\
ARDS pattern & $n=87$ & $n=63$ & $n=24$ \\
\hline CT scan & $63(72 \%)$ & $55(87 \%)$ & $8(33 \%)$ \\
\hline COVID-19 not suggestive & $13(15 \%)$ & $7(11 \%)$ & $6(25 \%)$ \\
Not typical for COVID-19 & $11(13 \%)$ & $1(1.6 \%)$ & $10(42 \%)$ \\
COVID-19 suggestive & & $p$ & $<0.001$ \\
\hline
\end{tabular}

LUS Lung ultrasound; $C T$ computed tomography; COVID-19 SARS-CoV-2 infection disease

All $p$ values $<0.001$

significantly associated with final COVID-19 diagnosis (86\% in COVID-19 vs $29 \%$ in non-COVID- $19, p<0.001$ ). Sensitivity, specificity, positive and negative predictive values and accuracy of each diagnostic testing in the entire study population and in different severity subgroups is reported in Table 6.
Fifteen $(83 \%)$ patients with false-negative RT-PCR in ED had a COVID-19-suggestive pattern at LUS, among them $10(56 \%)$ showed a typical ARDS pattern. Six $(33 \%)$ patients with false-negative RT-PCR in ED performed chest CT scan, of whom two (33\%) did not show COVID19 suggestive CT alterations. The presence in ED of at least one of positive SARS-CoV-2 naso-pharyngeal swab OR a

Table 6 Sensitivity, specificity, positive and negative predictive values and accuracy of diagnostic testing for COVID-19 (\%, $95 \mathrm{CI} \%$ )

\begin{tabular}{|c|c|c|c|}
\hline PoCUS & $\begin{array}{l}\text { Total } \\
n=360\end{array}$ & $\begin{array}{l}\text { Nonsevere } \\
n=164\end{array}$ & $\begin{array}{l}\text { Severe or critical } \\
n=196\end{array}$ \\
\hline Sensitivity & $86 \%(80-91 \%)$ & $70 \%(56-84 \%)$ & $92 \%(87-97 \%)$ \\
\hline Specificity & $71 \%(65-77 \%)$ & $84 \%(77-90 \%)$ & $55 \%(45-65 \%)$ \\
\hline PPV & $65 \%(58-72 \%)$ & $58 \%(49-67 \%)$ & $68 \%(56-80 \%)$ \\
\hline NPV & $89 \%(84-93 \%)$ & $90 \%(81-98 \%)$ & $87 \%(81-93 \%)$ \\
\hline Accuracy & $77 \%$ & $80 \%$ & $74 \%$ \\
\hline Chest CT scan & $n=87$ & $n=28$ & $n=59$ \\
\hline Sensitivity & $67 \%(48-86 \%)$ & $67 \%(29-100 \%)$ & $67 \%(45-84 \%)$ \\
\hline Specificity & $86 \%(77-94 \%)$ & $100 \%$ & $80 \%(68-93 \%)$ \\
\hline PPV & $64 \%(52-76 \%)$ & $100 \%$ & $60 \%(45-75 \%)$ \\
\hline NPV & $87 \%(74-100 \%)$ & $92 \%(65-100 \%)$ & $85 \%(69-100 \%)$ \\
\hline Accuracy & $80 \%$ & $93 \%$ & $75 \%$ \\
\hline RT-PCR ${ }^{a}$ & $n=360$ & $n=104$ & $n=256$ \\
\hline Sensitivity & $85 \%(79-91 \%)$ & $80 \%(68-92 \%)$ & $87 \%(80-94 \%)$ \\
\hline Specificity & - & - & - \\
\hline PPV & - & - & - \\
\hline NPV & $94 \%(86-96 \%)$ & $94 \%(86-100 \%)$ & $88 \%(81-95 \%)$ \\
\hline Accuracy & $94 \%$ & $95 \%$ & $93 \%$ \\
\hline
\end{tabular}

PoCUS Point-of-care ultrasound; $P P V$ positive predictive value; $N P V$ negative predictive value; $C T$ computed tomography; $R T-P C R$ reverse transcription polymerase chain reaction

${ }^{\text {a } O n ~ n a s o-p h a r y n g e a l ~ s w a b ~}$ 
COVID-19-suggestive LUS showed a sensitivity of $97 \%$ (95\% CI 94-100\%) and a NPV of 98\% (95\% CI 96-100\%). In severely symptomatic patients, combined LUS/swab sensitivity was $99 \%$ (95\% CI 97-100\%) and NPV was $98 \%$ (95\%CI 96-100\%). In patients with known SARS-CoV-2 exposition in the last 14 days, a COVID-19-suggestive pattern at LUS showed a PPV of 97\% (95\% CI 90-100\%) for COVID-19 diagnosis.

\section{Discussion}

From March to May 2020, SARS-CoV-2 spread widely through Italy, requiring a massive re-planning of ED, Hospital and National Health System organization. The main complaints were answering to the sudden lack of resources (in terms of individual protection devices, hospital admission capability and intensive care unit capability), and avoiding nosocomial SARS-CoV-2 spreading. A reliable tool to select which patients needed hospital isolation was critically important, especially for patients needing intensive or subintensive care. RT-PCR on respiratory tract swabs, despite being the gold standard for the diagnosis, has insufficient sensitivity to rule out SARS-CoV-2 infection, as multiple false-negative swabs are reported even in severely ill patients [29]. Moreover, in first-level EDs multiple RT-PCR testing actually requires a long process time: every test swab has to be sent to the hub centre for analysis, and actually the machine process time lasts hours. During this time, patients need to be kept in ED, resulting in increasing number of patients simultaneously present, otherwise they has to be sent in a "COVID-19-like" ward waiting for a definitive diagnosis. During SARS-CoV-2 or seasonal flu outbreaks a high number of COVID-19-like patients in ED is challenging for preventing nosocomial spreading and affecting other urgent ED activities.

In our study, PoCUS was reliable and reproducible in identifying patients with COVID-19, allowing its use in ED together with RCT-PCR for early identification of SARSCoV-2 infection in patients with suspect features. COVID-19 LUS findings are well described in previous literature $[8,13$, 15]. As COVID-19 manifestations range from asymptomatic infection to ARDS, a large number of COVID-19 patients could not show typical LUS findings. COVID-19 lung involvement typically starts from peripheral areas, allowing LUS to detect the disease even in the early stage. In our study population, only $61 \%$ of COVID- 19 patients showed a typical ARDS pattern at LUS. We defined a more sensitive LUS interpretation based on basic and easily identifiable findings for COVID-19 diagnosys. In our study, PoCUS showed a good sensitivity for COVID-19 during a SARS$\mathrm{CoV}-2$ outbreak and improved the diagnostic yield in ED.
Currently, the standard of care for early COVID-19like patients identification is chest CT scan. Chest CT scan demonstrated to be sensitive and specific [3]. Nevertheless PoCUS has the advantages to be widely available, reproducible and non-invasive and could be routinely used for first-line diagnostic stratification in ED together with RTPCR. The logistical difficulties related to the use of CT scan are reduced or even eliminated with PoCUS: patients can be evaluated bedside avoiding movement from and to the COVID pathway, device sanitization is fast and cheap, allowing the execution of a large number of test without affecting other ED activities, and the absence of ionizing radiations make even repeated evaluation safe also in young patients. In our study, PoCUS proved to have a high diagnostic accuracy and to be helpful in early identification of patients that needed to be managed in COVID-19-like areas even in presence of a negative RT-PCR testing in a nonselected COVID-suspected population in ED. That was particularly true in patients that needed hospitalization. Combined naso-pharyngeal swab and PoCUS sensitivity in critical patients was 99\%. Mild COVID-19 patients without clinical pneumonia and with low oral viral load are still very challenging to be identified with current testing possibilities. Non-severely ill patients needing hospital admission, especially if hypoxic, often show lung involvement that can be quickly detected by LUS. In our study, LUS was able to detect most patients with false-negative RT-PCR, empowering the diagnostic yield of ED evaluation. This could allow EPs to perform chest CT scan in selected patients with difficult or non diagnostic PoCUS only, with considerable sparing of time, money and viral contamination risk. In our study population, chest $\mathrm{CT}$ scan was performed according to EP judgement only in unclear clinical cases, generating a selection bias that prevents chest CT scan diagnostic yield evaluation. Anyway, a relevant percentage of nonconclusive $(13 / 87,15 \%)$ and false-negative $(8 / 24,33 \%)$ were found also in CT scan.

In most Italian hospitals, patients referring to ED must have a negative SARS-CoV-2 naso-pharyngeal swab before being accepted in any hospital ward [29]. During respiratory virus spreading seasons patients waiting for SARS-CoV-2 RT-PCR swab result can cause ED overcrowding, making it difficult to maintain patients distancing and avoiding nosocomial SARS-CoV-2 (as other airborne infectious diseases) spreading. According to our results, during a SARS-CoV-2 outbreak, patients with suggestive LUS findings cannot be ruled out for COVID-19 even in the occurrence of a negative RT-PCR in ED. These patients could be admitted in COVID19-like ward until RT-PCR and wait for further testing results, reducing ED process time and preventing overcrowding. This is particularly true for patients who need intensive care: critical and mechanically ventilated patients waiting for SARS-CoV-2 swab usually need to continue medical and nurse care and 
could be challenging for a low-resource setting. In our study population, severely ill patients COVID-19 suggestive PoCUS during a SARS-CoV-2 outbreak is suspicious for COVID-19 (PPV 68\%, 95\%CI 56-80\%).

\section{Limits}

Despite the prospective enrollment, not every COVID19-suspected patient could be evaluated with LUS in ED, causing a possible selection bias.

COVID-19 diagnosis is actually accepted only after demonstration of SARS-CoV-2 infection at RT-PCR, even in presence of strongly suggestive clinical and imaging patterns [23]. Due to the suboptimal sensitivity of RT-PCR, some patients with SARS-CoV-2 infection could result negatively even on multiple swab testing, overestimating other diagnostic test sensitivity and NPV of other diagnostic tests, as PoCUS. A Hawthorne-like effect could affect mild cases analysis due to possible less further testing. In our study LUS were performed by physicians with LUS expertyze. Even if LUS has a quick learning curve [14], beginners' exams could show a lower diagnostic accuracy. We described the accuracy of ED diagnostic test combinations comprehending RT-PCR itself to reproduce real ED diagnostic pathways. The presence of RT-PCR in the diagnostic pathway and the absence of a reliable diagnostic test as gold standard could probably overestimate the real diagnostic accuracy (incorporation bias). We found a significant difference in cardiovascular diseases among COVID and non-COVID subgroups ( $16 \%$ in COVID vs $6.4 \%$ in non-COVID, $p=0.01$ ). As at the best of our knowledge actually there is no data in literature suggesting a different exposure or different SARS-CoV-2 infection resistance in these subgroups or according to ongoing treatments, we assume that this could be related to an unintentional selection bias. Due to the possibility of lung ultrasound alteration in patients with previous cardiovascular diseases, this statistical abnormality could have affected our sensitivity analysis in this subset of patients.

\section{Conclusion}

PoCUS is a valuable tool for diagnostic stratification during COVID-19 outbreaks. LUS can help physicians in identifying false-negative RT-PCR, improving its diagnostic sensitivity in ED.

\section{Availability of data and material}

The datasets generated during and/or analysed during the current study are available from the corresponding author on reasonable request.
Author contributions All authors contributed to the study conception and design. Patient enrollment was performed by Caterina Savinelli, Camilla Tozzi, Elisa Paolucci and Michele Buggea. Chest CT scan revision and interpretation was performed by Germana Allescia. Patients follow-up was performed by Erica Sibona and Natalia Fersini. Data collection was performed by Lorenzo Pelagatti. Simone Bianchi, Diana Paolini and Michele Lanigra designed the study, performed literature search and selected, performed and interpreted statistical analyses and computations. The first draft of the manuscript was written by Simone Bianchi and Diana Paolini and all authors commented on previous versions of the manuscript. All authors read and approved the final manuscript.

Funding None obtained.

\section{Compliance with ethical standards}

Conflict of interest The authors declare that they have no conflict of interest.

Ethics approval The study was conducted in accordance with the 1964 Helsinki declaration and its later amendments, and was approved by the institutional review board.

Consent to participate and consent for publication Each patient gave freely given, informed consent to participate in the study and for publication of personal data. A legal tutor agreement was achieved for patients legally or physically unable to give informed consent.

\section{References}

1. Huang C, Wang Y, Li X et al (2020) Clinical features of patients infected with 2019 novel coronavirus in Wuhan, China [published correction appears in Lancet. 2020 Jan 30]. Lancet 395(10223):497-506. https://doi.org/10.1016/S0140-6736(20) 30183-5

2. Chen N, Zhou M, Dong X et al (2020) Epidemiological and clinical characteristics of 99 cases of 2019 novel coronavirus pneumonia in Wuhan, China: a descriptive study. Lancet 395(10223):507513. https://doi.org/10.1016/S0140-6736(20)30211-7

3. Ai T, Yang Z, Hou H et al (2020) Correlation of chest CT and RTPCR testing for coronavirus disease 2019 (COVID-19) in China: a report of 1014 cases. Radiology 296(2):E32-E40. https://doi.org/ 10.1148/radiol.2020200642

4. Wang W, Xu Y, Gao R et al (2020) Detection of SARS-CoV-2 in different types of clinical specimens. JAMA 323(18):1843-1844. https://doi.org/10.1001/jama.2020.3786

5. Kucirka LM, Lauer SA, Laeyendecker O et al (2020) Variation in false-negative rate of reverse transcriptase polymerase chain reaction-based SARS-CoV-2 tests by time since exposure. Ann Intern Med. https://doi.org/10.7326/M20-1495

6. Xie X, Zhong Z, Zhao W, Zheng C, Wang F, Liu J (2020) Chest CT for typical $2019 \mathrm{nCoV}$ pneumonia: relationship to negative RT-PCR testing. Radiology. https://doi.org/10.1148/radiol.20202 00343

7. Huang P, Liu T, Huang L, Liu H, Lei M, Xu W et al (2020) Use of chest CT in combination with negative RT-PCR assay for the 2019 novel coronavirus but high clinical suspicion. Radiology 295:22-23. https://doi.org/10.1148/radiol.2020200330

8. Pata D, Valentini P, De Rose C, De Santis R, Morello R, Buonsenso D (2020) Chest computed tomography and lung ultrasound findings in COVID-19 pneumonia: a pocket review for 
non-radiologists. Front Med (Lausanne) 7:375. https://doi.org/ 10.3389/fmed.2020.00375

9. Marco A, Alberto P, Martina G et al (2020) Lung ultrasound may support diagnosis and monitoring of COVID-19 pneumonia. Ultrasound Med Biol. https://doi.org/10.1016/j.ultrasmedbio. 2020.07.018

10 Zhao W, Zhong Z, Xie X, Yu Q, Liu J (2020) Relation between chest $\mathrm{CT}$ findings and clinical conditions of coronavirus disease (COVID-19) pneumonia: a multicenter study. AJR Am J Roentgenol. 214(5):1072-1077. https://doi.org/10.2214/AJR.20.22976

11 Bai HX, Hsieh B, Xiong Z et al (2020) Performance of radiologists in differentiating COVID-19 from non-COVID-19 viral pneumonia at chest CT. Radiology 296(2):E46-E54. https://doi. org/10.1148/radiol.2020200823

12 Chung M, Bernheim A, Mei X et al (2020) CT imaging features of 2019 novel coronavirus (2019-nCoV). Radiology 295(1):202207. https://doi.org/10.1148/radiol.2020200230

13. Soldati G, Smargiassi A, Inchingolo R et al (2020) Is there a role for lung ultrasound during the COVID-19 pandemic. J Ultrasound Med. https://doi.org/10.1002/jum.15284

14 Mayo PH, Copetti R, Feller-Kopman D et al (2020) Thoracic ultrasonography: a narrative review. Int Care Med. https://doi.org/10. 1007/s00134-019-05725-8

15. Soldati G, Smargiassi A, Inchingolo R et al (2020) Proposal for international standardization of the use of lung ultrasound for patients with COVID-19: a simple, quantitative, reproducible method. J Ultrasound Med. https://doi.org/10.1002/jum.15285

16 Wang W, Xu Y, Gao R et al (2020) Detection of SARS-coV-2 in different types of clinical specimens. JAMA. https://doi.org/10. 1001/jama.2020.3786

17. Soldati G, Smargiassi A, Inchingolo R et al (2020) On Lung Ultrasound patterns specificity in the management of COVID-19 patients. J Ultrasound Med. https://doi.org/10.1002/jum.15326. 10.1002/jum. 15326

18. Smith MJ, Hayward SA, Innes SM, Miller ASC (2020) Pointof-care lung ultrasound in patients with COVID-19-a narrative review. Anaesthesia 75(8):1096-1104. https://doi.org/10.1111/ anae. 15082

19. DGRT n. 669 del 18-06-2018. Allegato A "Modello organizzativo dell'Emergenza Pediatrica Ospedaliera. Linee di indirizzo." (2018) http://www.quotidianosanita.it/allegati/allegato3489968. pdf

20. DGRT n.1380/2016 "Reti cliniche tempo-dipendenti di cui al D.M. 70/2015. Approvazione documenti di programmazione delle reti regionali." Allegato C. (2016) https://www.regione.toscana. it/bancadati/atti/Contenuto.xml $?$ id $=5135888 \&$ nomeFile $=$ Delib era_n.1380_del_27-12-2016-Allegato-C

21. World Health Organization (2020) Global surveillance for COVID-19 caused by human infection with COVID-19 virus: interim guidance. 20 March 2020. WHO/2019-nCoV/ SurveillanceGuidance/2020.6

22. World Health Organization (2020) Laboratory testing of human suspected cases of novel coronavirus (nCOV) infection: interim guidance January 12, 2020. https://apps.who.int/iris/bitstream/ handle/10665/330374/WHO-2019-nCoV-laboratory-2020.1-eng. pdf

23. Ministero della Salute (2016) Ministero della Salute-Assistenza, ospedale e territorio-118 e Pronto Soccorso-I codici colore gravità (triage). Ref type: online source

24. Lipley N (2005) Updated Manchester triage system published this month. Emerg Nurse 13(7):3

25. Accademia di Ecografia Toracica-Pleural Hubbers Team (2019) COVID-19 MANAGEMENT poster. https://www.ordine-medicifirenze.it/modulistica-docman/varie/311-adet/file. Ref type: online source

26. World Health Organization (2020) Clinical management of COVID-19. Interim guidance 27 May 2020. WHO/2019-nCoV/ clinical/2020.5

27. Chung M, Bernheim A, Mei X, Zhang N, Huang X, Xu W et al (2019) CT imaging features of novel coronavirus (2019-nCoV). Radiology 295(1):202-207. https://doi.org/10.1148/radiol.20202 00230

28. Società Italiana di Emergenza Urgenza (SIMEU) (2019) Rapporto Prima Linea COVID-19_ecografia. https://www.simeu.it/w/artic oli/leggiArticolo/4031/leggi. Ref type: online source

29. http://www301.regione.toscana.it/bancadati/atti/Contenuto.xml? id $=5247300 \&$ nomeFile $=$ Ordinanza_del_Presidente_n.14_del_ 17-03-2020-Allegato-A (2020). Ref type: online source

30. Siddiqi HK, Mandeep RM (2020) COVID-19 illness in native and immunosuppressed states: a clinical-therapeutic staging proposal. J Heart Lung Transplant 39(5):405-407. https://doi.org/10.1016/j. healun.2020.03.012

Publisher's Note Springer Nature remains neutral with regard to jurisdictional claims in published maps and institutional affiliations. 